Horizons philosophiques

\title{
Réalité et virtualité de la réalité virtuelle
}

\section{Adriano P. Palma}

Volume 6, numéro 2, printemps 1996

La philosophie sur Internet

URI : https://id.erudit.org/iderudit/801010ar

DOI : https://doi.org/10.7202/801010ar

Aller au sommaire du numéro

\section{Éditeur(s)}

Collège Édouard-Montpetit

\section{ISSN}

1181-9227 (imprimé)

1920-2954 (numérique)

Découvrir la revue

\section{Citer cet article}

Palma, A. P. (1996). Réalité et virtualité de la réalité virtuelle. Horizons philosophiques, 6(2), 55-59. https://doi.org/10.7202/801010ar d'utilisation que vous pouvez consulter en ligne.

https://apropos.erudit.org/fr/usagers/politique-dutilisation/ 


\section{RÉALITÉ ET VIRTUALITÉ DE LA RÉALITÉ VIRTUELLE}

\section{Ce qui nous sépare de la réalité virtuelle}

En dépit de tout ce qui a pu se dire au sujet de l'autoroute électronique, nous sommes encore loin de vivre dans un environnement qui se laisserait désigner comme réseau global. Àl'heure actuelle, le nombre d'individus branchés sur la planète reste infime, à l'exception des États-Unis. Ailleurs, ceux qui voudraient éviter la routine fastidieuse consistant à digitaliser l'information au moyen d'un traitement de texte, pour ensuite l'imprimerpuis l'expédier aumoyen d'un télécopieur (qui digitalise l'information pour l'expédier vers un autre télécopieur, qui produit une nouvelle copie imprimée), se heurtent à une foule de problèmes, qui ont pour cause principale le retard technologique. En termes d'infrastructures et de compatibilité, nous sommes loin du fameux village global. Des contrées relativement développées n'ont pas les installations nécessaires aux réseaux informatiques. Sans parler des problèmes culturels. La simple existence de formes de connexions qui échappent aux contrôles traditionnels est problématique pour les mentalités tatillonnes avides de censure. Ainsi, pour ne citer qu'un exemple, certains sites restreignent l'accès aux FTP (File Transfer Protocol, transfert de fichiers entre deux systèmes), TELNET et autres utilitaires, en vue de protéger les droits d'auteur ou les secrets militaires. Les difficultés viennent surtout de ceux qui tout en ayant une culture informatique, considèrent encore l'ordinateur comme une machine à écrire sophistiquée. Un message diffusé sur Internet est-il une communication en bonne et due forme? La question n'est pas sans importance du point de vue bureaucratique. Le désespoir financier, les lignes téléphoniques défaillantes et le quasi monopole de l'État sur les télécommunications s'allient le plus souvent avec la culture du papier pour s'opposer à ce que la réalité virtuelle devienne une réalité concrète. 
La seule opposition véritable aux forces de réaction provient de la nature même du processus que nous appelons "réalité virtuelle". C'est elle qui explique que les réseaux aient continué de s'étendre. L'Internet rend disponibles des masses d'informations, dont peuvent profiter les contrées du TiersMonde (et du deuxième et demi), où le prix des publications imprimées les rend souvent complètement inaccessibles. Mais la réalité virtuelle s'imposera progressivement, à mesure que l'on fera taire les arguments conservateurs portant sur le coût des investissements nécessaires au branchement, relativement à d'autres méthodes de partage de l'information qui sont en fait complètement dépassées.

\section{La fragmentation du savoir}

Quel est l'impact de l'existence des réseaux sur les humanités? II semble que celles-ci pratiquent de plus en plus un style d'intervention qui les rapproche des sciences "pures» d'antan. Il s'agit de moins en moins de présenter un système, ce qui est encore la forme typique du livre. Ce qui est de plus en plus populaire, ce sont les remarques incisives, qui présentent brièvement les résultats de formalisations antérieures.

Dans la tradition humaniste, le livre était ce qui permettait à son auteur de se présenter comme érudit maîtrisant un corpus de textes, auquel il venait ajouter son grain de sel critique. Si on jette un coup d'œil sur l'histoire des humanités, une des caractéristiques principales de leur développement est qu'on doit toujours à nouveau repartir à zéro. Chaque penseur se croit obligé de remettre le monde à l'endroit. Or l'extension des réseaux à la communauté des professionnels œuvrant dans le domaine des humanités a entraîné une fragmentation du savoir. Les sous-disciplines se multiplient sans vraiment avoir quoi que ce soit de commun entre elles. Les humanités approchent ainsi de l'état de "sciences matures", suivant la classification de Kuhn. Pour ces sciences, travailler consiste à résoudre des problèmes qui relèvent des paradigmes de ces sciences ellesmêmes. Dans ces conditions, une révolution ne saurait plus se produire (on a tout au plus une discipline qui se détache de sa 
racine humaniste, comme ce fut le cas de la linguistique, à l'occasion de la «révolution» opérée parChomsky), et le jugement final sur la situation doit rester ouvert. On peut y voir le signe d'une stabilisation relative des humanités en tant que disciplines closes sur elles-mêmes, ce qui les rendrait complètement inutiles, ou on peut y voir le terminus ad quem d'un processus de spécialisation des sciences humaines qui est en lui-même l'indice de leur maturité.

\section{La philosophie comme entre-deux}

Le problème de la fragmentation du savoir intéresse au premier chef la philosophie. L'agora est plus large que jamais, traversant les frontières nationales. Les interventions sont plus libres que dans le milieu universitaire. Les étudiants sont enfin libres de poser les fameuses questions que chacun voulait poser sans oser le faire. Évidemment, le niveau général s'en ressent mais le bon côté de la chose est qu'on assiste au retour de la philosophie sérieuse à l'ancienne. En effet, la méthode socratique ne revenait-elle pas à poser des questions sur les points qui paraîtraient trop évidents pour mériter un examen? Orc'est ce qui se produit de plus en plus surl'Internet. De petites questions sont posées, parfois légèrement, entraînant à leur suite une foule d'exemples et de contre-exemples, de tentatives d'explication qui sont loin de faire l'unanimité, même si elles finissent par nous rappeler que le soleil se couche et qu'il est temps de délaisser les affaires publiques pour les soucis domestiques.

II ne faut pas oublier que l'on ne saurait fournir qu'un tableau imaginaire d'événements qui se produisent dans de vastes secteurs de la communauté philosophique. Néanmoins, il est remarquable à quel point de simples changements technologiques entraînent inéluctablement des changements de mentalité. Ainsi, le passage de l'esprit de système à la remarque incisive souligné plus haut n'est pas sans effet sur la philosophie. Car celle-ci se voit ramenée à son état de chaos primitif. 


\section{Ordre et chaos ou les effets internes de la réalité virtuelle sur la philosophie}

La réalité virtuelle affecte-t-elle la philosophie de manière interne? Un effet interne porterait non seulement sur les modes de production de la philosophie mais sur les thèses qui sont avancées et débattues. Or l'usage montre la vérité de l'adage médiéval «in corpore vili». L'ordre peut surgir d'interactions chaotiques, le "chaos» étant compris dans son sens technique aussi bien qu'ordinaire. Les discussions peuvent partir des questions les plus terre à terre (comment enseigner la sémantique de vérité des propositions conditionnelles dans un cours d'introduction à la logique) pour aboutir très tôt à un point où des questions très sérieuses seront débattues. On a ainsi pu observer, sur une des listes de logique, une discussion qui partant des fonctions de vérité s'est par la suite intéressée à la comparaison entre les performances de sujets non éduqués en logique et celles d'individus compétents dans cette matière, sur la question des problèmes de choix de Wason'. La question peut paraître ésotérique, mais elle va droit au cœur du lien entre la logique comme discipline formelle et la faculté humaine naturelle de raisonner.

Voici ce qui est montré par cet exemple et bien d'autres. Depuis longtemps, nous pensons la pensée comme si elle était enfermée à l'intérieur de la personne, où la direction de l'intellect obéit à des règles. Or ce que démontre la philosophie sur l'Internet est que nous n'avons pas besoin de règles. Les interactions peuvent être aussi chaotiques que l'on voudra, elles finissent par atteindre un certain niveau d'intérêt ou elles meurent dans une espèce de processus évolutif. La supériorité des modèles connexionistes sur les modèles cognitifs fondés sur des règles, pour ce qui est de décrire le fonctionnement de la pensée, ne se trouve pas démontrée par là. Toutefois, ceux qui estiment que la philosophie de l'esprit ou «philosophy of mind" devrait tenir compte du fait que la pensée n'est pas individuelle, contrairementà notre corps, auront une plus grande

1. II s'agit de tester l'aptitude à appliquer des règles. 
crédibilité. De même, l'émergence d'un ordre ou de ce qui s'en rapproche le plus pourrait être un coup de pouce dans le sens d'une sémantique naturelle plutôt que d'un système, dont les seules règles sont en fait des exigences purement syntaxiques. Et si quelque chose se montrait également dans le cas de l'éthique?

Adriano P. Palma (pyapp @ccunix.ccu.edu.tw) Institute of philosophy and COGSCl Center National Chung-Cheng University

Taïwan

Traduction de Josette Lanteigne 